\title{
EVALUATION OF RESULTS OF BRIDGE PLATING IN COMMINUTED FRACTURES OF LONG BONES
}

Prasanta Kr. Mandal, Fagu Ram Majhi, Dibakar Ray, Mrinal Kanti Roy, M. Bhattacharyya, Amalendu Bikas Chatterjee

\footnotetext{
1. Associate Professor. Department of Orthopedics, Bankura Sammilani Medical College.

2. Assistant Professor. Department of Orthopedics, Bankura Sammilani Medical College.

3. Assistant Professor. Department of Orthopedics, Bankura Sammilani Medical College.

4. Assistant Professor. Department of Orthopedics, Bankura Sammilani Medical College.

5. Professor. Department of Orthopedics, Bankura Sammilani Medical College.

6. Associate Professor. Department of Anaesthesia, Bankura Sammilani Medical College.
}

\section{CORRESPONDING AUTHOR:}

Prasanta Kr. Mandal

Lokepur, P. O. Kenduadihi, Dist.

Bankura, Pin- 722102.

E-mail: drprasantamondal@gmail.com

Ph: 009194341173124.

ABSTRACT: INTRODUCTION: Osteosynthesis with open reduction technique in comminuted fracture has several disadvantages like increase rate of non-union, soft tissue problem, infection and implant failure. These problems may be overcome by percutaneous bridge plating technique which preserve the vascularity and soft tissue attachment of fracture fragments. MATERIALS AND METHODS: Twenty-five patients were involve in this study . All of twentyfive patients with closed comminuted diaphyseal fractures of femur (n-10) and tibia (n-15) were operated on using the principle indirect reduction and biological fixation with bridge plate under image intensifier. Partial and full weight bearing were allowed according to clinical and radiological union. RESULTS: Most regained excellent range of motion of knee and ankle. Mean time of radiological union was 20.12 weeks. Two patients had superficial skin problem which healed with local wound care. One patient had non union which was healed by bone grafting. In this study excellent result was seen in 18 cases, good in five and fair in two patients. CONCLUSION: Our study indicates that minimally invasive bridge plating is an effective alternative for treatment of comminuted diaphyseal fracture of long bones. These complex fractures give complete recovery of normal limb function at an early stage with less complication.

KEYWORDS: Bridge plating, minimally invasive, diaphyseal fracture of long bone

INTRODUCTION: In our day to day life musculoskeletal injuries are increasing in number. Fixation of fracture with maximum preservation of surrounding soft tissue is the objective of all system of internal fixation. Nelson (1964) ${ }^{1}$ and Rhinelander (1968) ${ }^{2}$ belived that intramedullary blood supply is most important in normal bone healing. However after injury the intramedullary vascular pattern is disrupted and periosteal blood supply increases and becomes the chief sources of blood supply. So, while using the technique of internal fixation of long bone, emphasis must be on vascular support of bone and soft tissue by doing minimum exposure, indirect reduction of fracture fragments and in particular the minimal possible damage to the periosteal blood supply.

Clinical experience has shown that early AO/ASIF method of plating in comminuted fractures of long bones to ensure absolute stability and primary bone union has been associated 
with high risk of non-union. Recent experiences suggest that leaving the fragment untouched preserve their blood supply and accelerate union. Biological percutaneous plating provides relative stability, preserve vascularity around the fractures and it also allows controlled micromotion, resulting in rapid and more abundant callus formation, as observed in intramedullary nailing or in non operative fracture treatment. Thus bridge plating with minimum invasive technique has occupied the dominant position in recent advancement of treatment of comminuted fractures $3,4,5,6$.

The present study was undertaken in prospective basis to evaluate the efficacy of bridge plating in comminuted fracture of femur and tibia.

Materials and Methods

Between 2008 and 2011 we treated 25 patients of whom 10 had femoral fracture and 15 had tibial fractures. An inclusion criterion was adult patient with closed comminuted diaphyseal fractures of femur and tibia. Cases with simple fracture, pathological fracture and periprosthetic fracture were excluded.

Initial treatment considers skeletal traction for femoral fracture and plaster slab for tibial fracture. Patients were operated under general or spinal anaesthesia and tourniquet were applied whenever possible. A fracture table and traction were used for the femur fractures. An image intensifier was used to assess fracture alignment and progress of fixation. Fixation was accomplished using standard instrumentation and narrow and broad dynamic compression plate (DCP) implants instead of locking plate only because of cost effectiveness keeping in mind the difficultness to introduce the DCP beyond fracture site particularly in femoral fractures. After the fracture was manipulated and satisfactory alignment was achieved, through proximal and distal small incisions a tunnel was made submuscularly in femur and subcutaneous in tibia (fig. 1). A DCP implants were placed in the proximal and distal to fracture site. The plate was fixed with screws on either side with a purchase of minimum six cortices both above and below the fracture, under image guidance. Intermediate fragments were left undisturbed. The wound was closed in layers. All patients received prophylactic antibiotic for 7 days. An isometric quadriceps program was initiated from $3^{\text {rd }}$ post operative day and the patients were mobilized with the help of crutches on $7^{\text {th }}$ post operative day. Partial weight bearing was allowed when callus was observed at the fracture site in a single plane radiographic images and full weight bearing was started on observation of callus in two planes radiographic images. Patients were followed up at 4 weeks interval clinically and radiologically.

RESULTS AND ANALYSIS: Total no. Of patients was 25(22 male and 3 female) of whom 10 had femoral fracture and 15 had tibial fracture. Average age was 34years(most common group being 20-30 years). Road traffic accident was the major causes of these fractures. Most of the fractures were fixed internally within first 7 days of injury. Partial weight bearing was started with the help of crutches between 8- 13 weeks. Patients were followed up for a period of 12-48 months( average 24 months). Average time of radiological union was 20.12 weeks. In this study only one case of superficial infection and one case of skin necrosis was observed. Only one cases of nonunion was observed. Results of internal fixation were assessed according to criteria laid down by S.J. Lam (1964).

Excellent: Range of movement of adjacent joints $80-100 \%$ of normal. No pain in performing daily activities. 
Good: Range of movement of adjacent joints $60-80 \%$ of normal. Pain not enough to cause any modification of patient daily routine.

Fair: Range of movement of adjacent joints $30-60 \%$ of normal. Pain enough to cause restriction of patient's daily activities.

Poor: Range of movement of adjacent joints less than $30 \%$ of normal. Pain enough to cause severe disability or non-union.

DISCUSSION: The development of modern nailing techniques has improved the overall outcome and set new standards for operative treatment of fracture of long bones. Along with the evolution of nailing technique, there has been a similar evolution of plating technique. Clinical thinking has shifted from the mechanical concept of absolute stability to a more biological concept of indirect reduction with limited or no approach at the fracture zone and epiperiosteal plate application?

Radiological union (callus in two planes of radiographs) and clinical signs namely absence of pain and tenderness, were taken as criteria to allow full weight bearing. The average time of union was 20.12weeks. Several cases of fracture tibia (15 cases) complained of ankle and knee stiffness initially but after 3 weeks, restricted movement was seen only 3 cases. Overall incidence of ankle was $27 \%$, while in one patient (9\%) movement was 10 degree less than normal. Complication rate was very low in the present series of study. There was no evidence of deep infection. Only one case of non-union was observed. The excellent success rate was achieved due to the indirect reduction of fractures with axial and torsional alignment rather than perfect anatomical reduction $7,8,9$.

SUMMARY AND CONCLUSION: Present series consisted of 25 cases of comminuted diaphyseal fractures of tibia and femur. Among 25 patients, 15 had tibial fracture and 10 had femoral fracture. All were fixed with bridge plating with minimum incision without exposing the fracture site. The average age of the patients was 34 years with male preponderance and road traffic accident being the major cause of these high energy fractures as $28 \%$ of patients had associated fractures. Average time of radiological union in this series was 20.12 weeks ranging from 16 to 30 weeks.

The highlights of the present series are that there were only very few complications with no cases of deep infection and delayed union; overall excellent and good result were seen in $92 \%$ cases. This achievement could be attributed only to biological internal fixation of fracture with minimum handling of soft tissue along with relatively stable internal fixation.

From the present study we conclude that biological internal fixation of comminuted fractures of long bones by bridge plating has overcome the previous disadvantages of rigid and more invasive internal fixation techniques by plates or vascular damage caused by intramedullary nails. Biological fixation of complex fractures gives stable as well as optimal internal fixation and complete recovery of limb function at an early stage than by fixation by other methods with minimal risk of complications.

\section{REFERENCES:}

1. Gustilo RB; Nelson GE: The effect of intramedullary nailing on the blood supply of diaphysis of long bones in mature dogs. J.B.J.S. 46-A: 1362-1363, 1964 
2. Rhinelander WF; The normal microcirculation of diaphyseal cortex and its response to fracture.J.B.J.S.55A:895-899,1968.

3. Gerber C; Mast JW: Biological internal fixation of fractures. J. Orth. Trauma. 109:295303, 1990.

4. Baumgaertel F. Bridge plating, In; Ruedi TP, Murphy WM, eds AO Principles of fracture management. New York, NY: Thieme; 2000; 221-231.

5. Krettek C. Concepts of minimally invasive. Injury, 1997; 28(suppl)1-6.

6. Mohammad Javdan, Ali Andalib, Biological plating of comminuted fracture of femur and tibia, Journal and Research in medical Science, July and August 2007; Vol 12, No. 4; 186189.

7. Baumgaertel F, Buhl M, Rahn BA. Fracture healing in biological plate osteosynthesis. Injury 1998; 29(suppl);C3-C6

8. Sturmer KM. Elastic plate osteosynthesis, its biomechanics, indication and technique compared with rigid internal fixation. Unfallchirurg. 1996; 99;816-819.

9. Costas Papakostidis,MD; Martin R.W. Grotz,MD-Femoral biologic plate fixation. Clinical Orthopaedics and Related Research. No-450, September 2006

\section{Table-1 Showing functional results of the study}

\begin{tabular}{|l|l|l|}
\hline Criteria & No. Of patients & Percentage (\%) \\
\hline Excellent & 18 & 72 \\
\hline Good & 5 & 20 \\
\hline Fair & 2 & 8 \\
\hline Poor & - & - \\
\hline Total & 25 & 100 \\
\hline
\end{tabular}
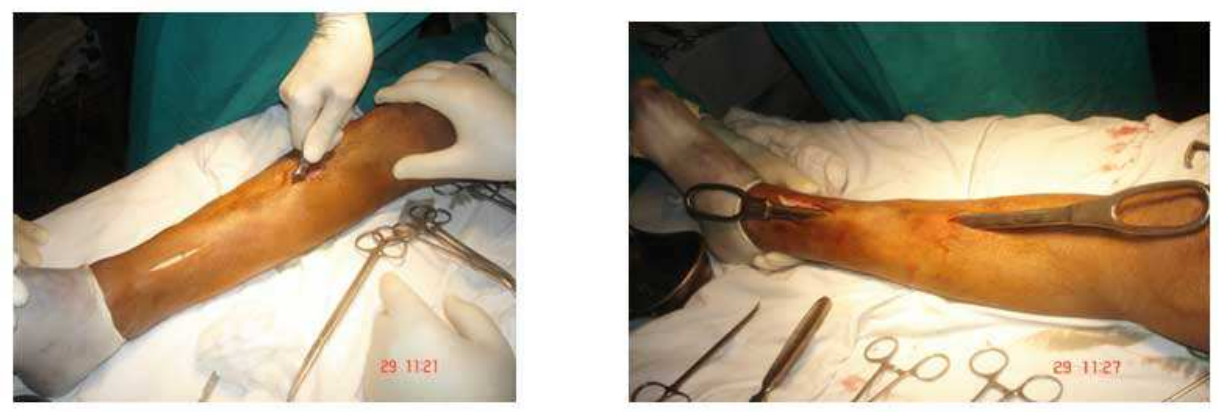

Fig 1 \&2: Extraperiosteal tunneling 


\section{ORIGINAL ARTICLE}

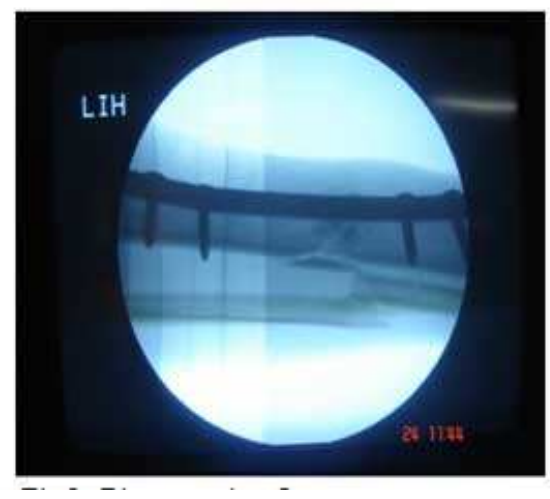

Fig3: Plate under C-arm
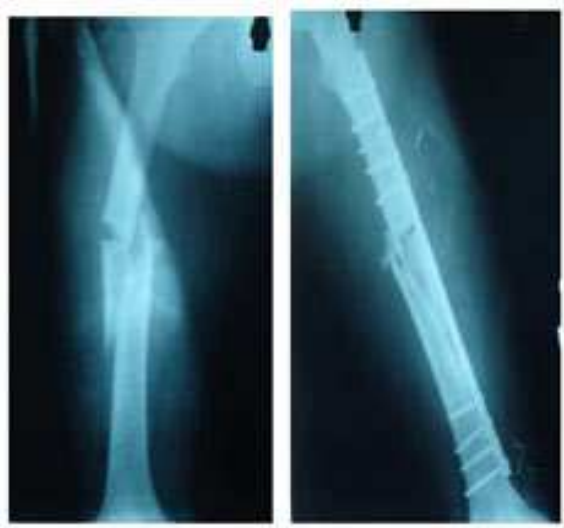

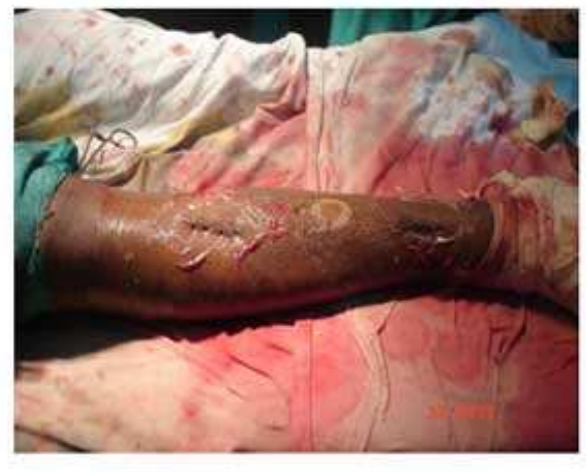

$-\cdots$

Fig4: After closure

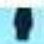

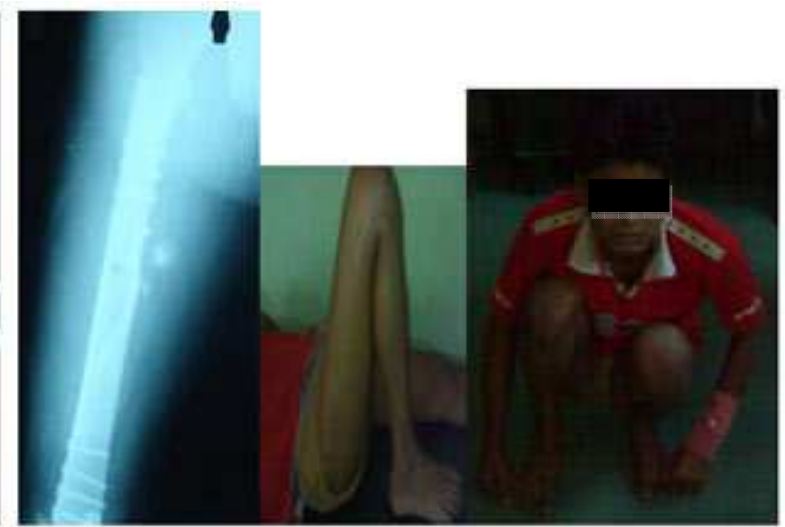

Fig 5a: Preoperative Fig5b:Immediate Postop Fig5c:12 weeks followup Fig5d \&5e: showing ROM of joint
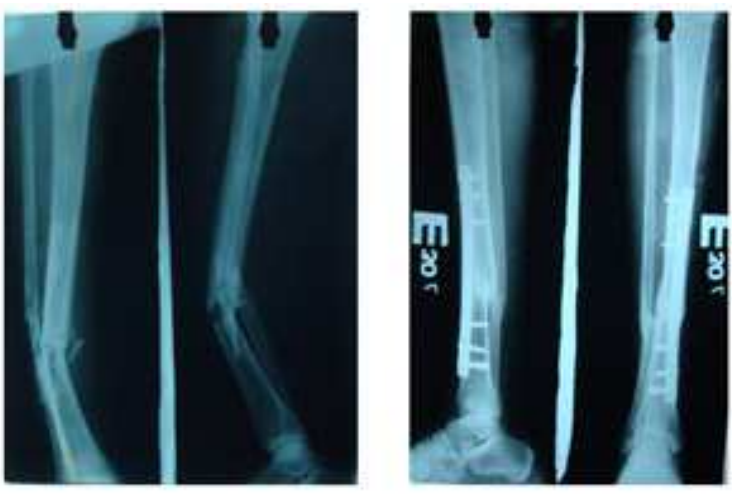

Fig6a: Preoperative x- ray Fig6b: at 16 weeks follow up
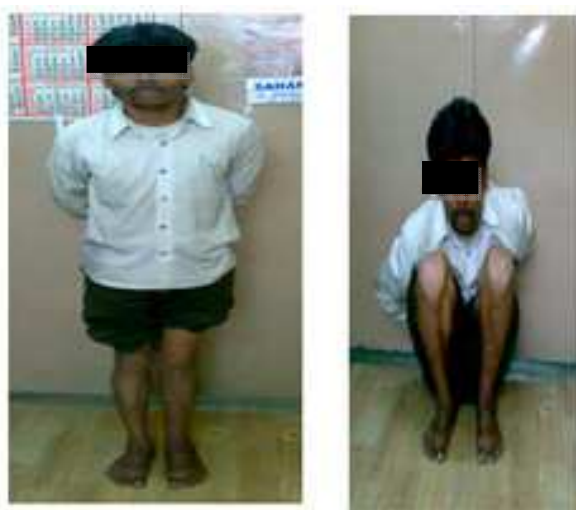

Fig6c \&6d : showing RangeOf Motion at follow up 


\section{ORIGINAL ARTICLE}

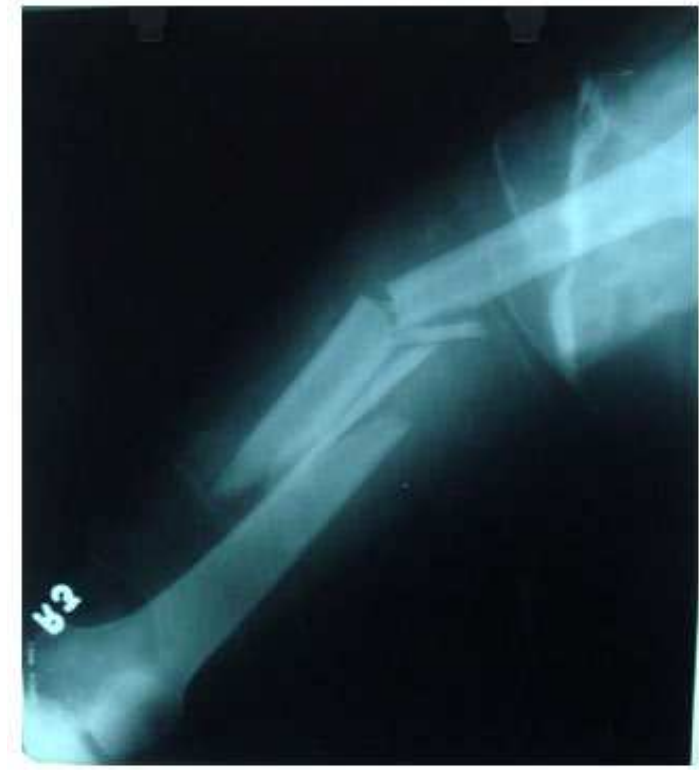

: Preoperative Xray

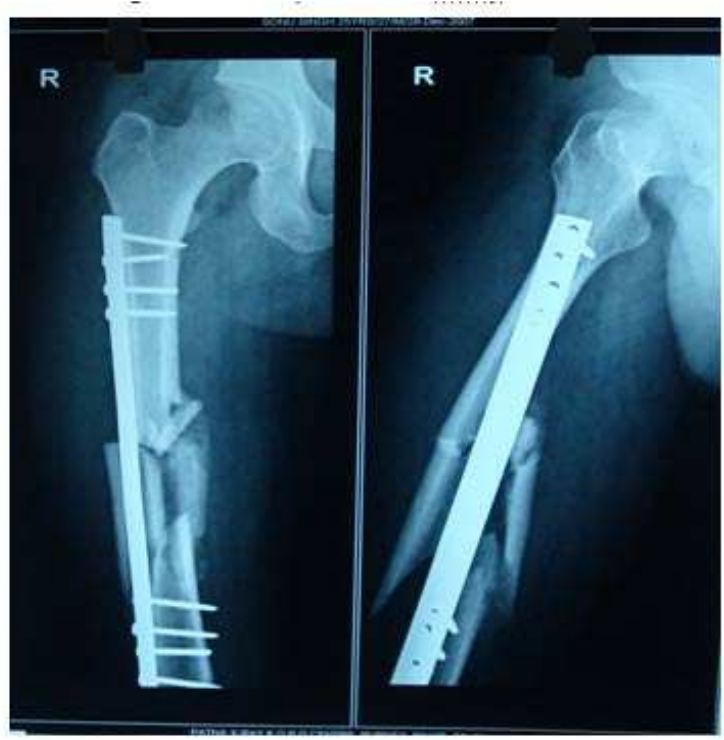

Fig 7c:12 weeks followup Xray

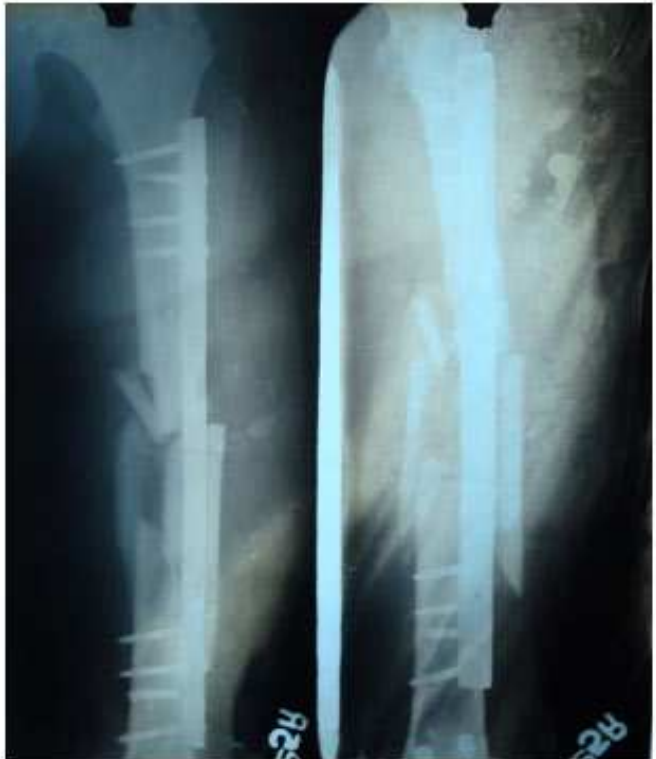

Fig7b:Immediate Postoperative Xray

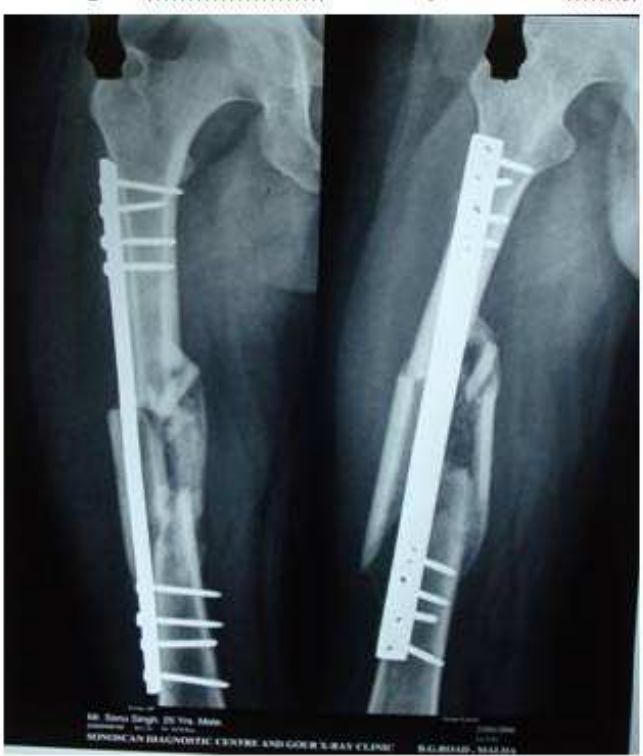

Fig7d: 22 weeks followup Xray 


\section{ORIGINAL ARTICLE}

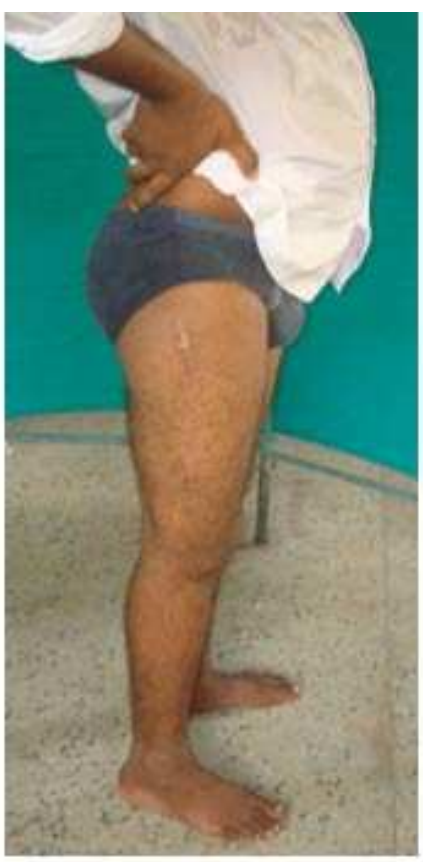

Fig 7e:

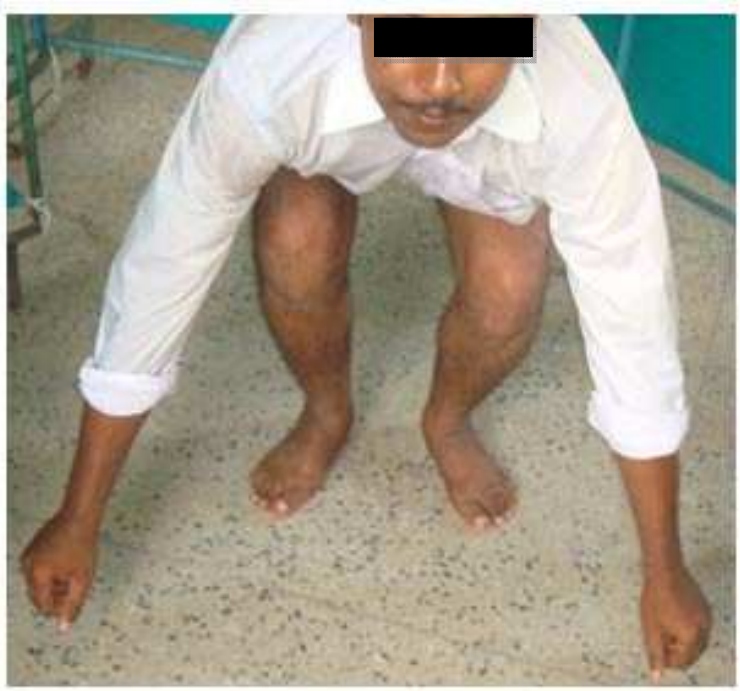

Fig 7f:

Fig 7e \&7f: showing range of motion at follow up 